\title{
Demonstrating the GaInP/GaAs Three-Terminal Heterojunction Bipolar Transistor Solar Cell
}

\author{
Marius H. Zehender, Iván García, Simon A. Svatek, Myles A. Steiner , Pablo García-Linares , Emily Warren , \\ Adele Tamboli Antonio Martí, Elisa Antolín
}

\begin{abstract}
The three-terminal heterojunction bipolar transistor solar cell (HBTSC) concept enables the realization of a monolithic double-junction device with individual current extraction. We present an HBTSC realized by a heterojunction of GaInP and GaAs. The one-sun open-circuit voltage $\left(V_{O C}\right)$ of the top and bottom junctions are $1.33 \mathrm{~V}$ and $0.99 \mathrm{~V}$, respectively, while fill factors $(F F)$ are above $80 \%$. At one-sun illumination, reducing one junction's bias from $V_{O C}$ to maximum power point degrades the performance of the other junction only slightly $(<$ $0.5 \%$ efficiency loss). These results demonstrate the potential of the HBTSC concept to produce high-efficiency independently connected double-junction solar cells.

Index Terms-double junction, multi terminal, independent current, photovoltaic cells, gallium indium phosphide, gallium arsenide
\end{abstract}

\section{INTRODUCTION}

In the most common implementation of multijunction solar cells, junctions are connected in series. The classical approach to reach higher efficiencies is to increase the number of junctions connected in series. Even if the effect of the series connection on the absolute efficiency limit is relatively small, for three junctions or more there is a strong spectral sensitivity and the annual energy yield of series-connected devices is affected by spectral mismatch losses [1]. In fact, even in the case of optimum bandgaps, switching to an independent connection of the junctions results in a higher increase in annual energy efficiency than adding a fourth junction [2]. Furthermore, independently connected multijunction solar cells are less sensitive to non-optimum bandgaps of the materials than series-connected multijunction solar cells. Therefore, the pool of materials to choose from for a high efficiency multijunction cell is larger and also bandgap variations due to temperature have a lesser effect. For these reasons, there is an increasing interest in developing independent current extraction using different approaches [3]-[7].

A recently proposed concept is the three-terminal heterojunction bipolar transistor solar cell (HBTSC) [3], which besides enabling independent current extraction, has the advantage of a very simple structure: only three basic semiconductor layers are necessary. In this work, we demonstrate a monolithic III-V semiconductor HBTSC made of only six layers, including contact, window and back surface field (BSF) layers.

The HBTSC has a design similar to the design of a bipolar transistor. It can be realized with a combination of $n-p-n$ or

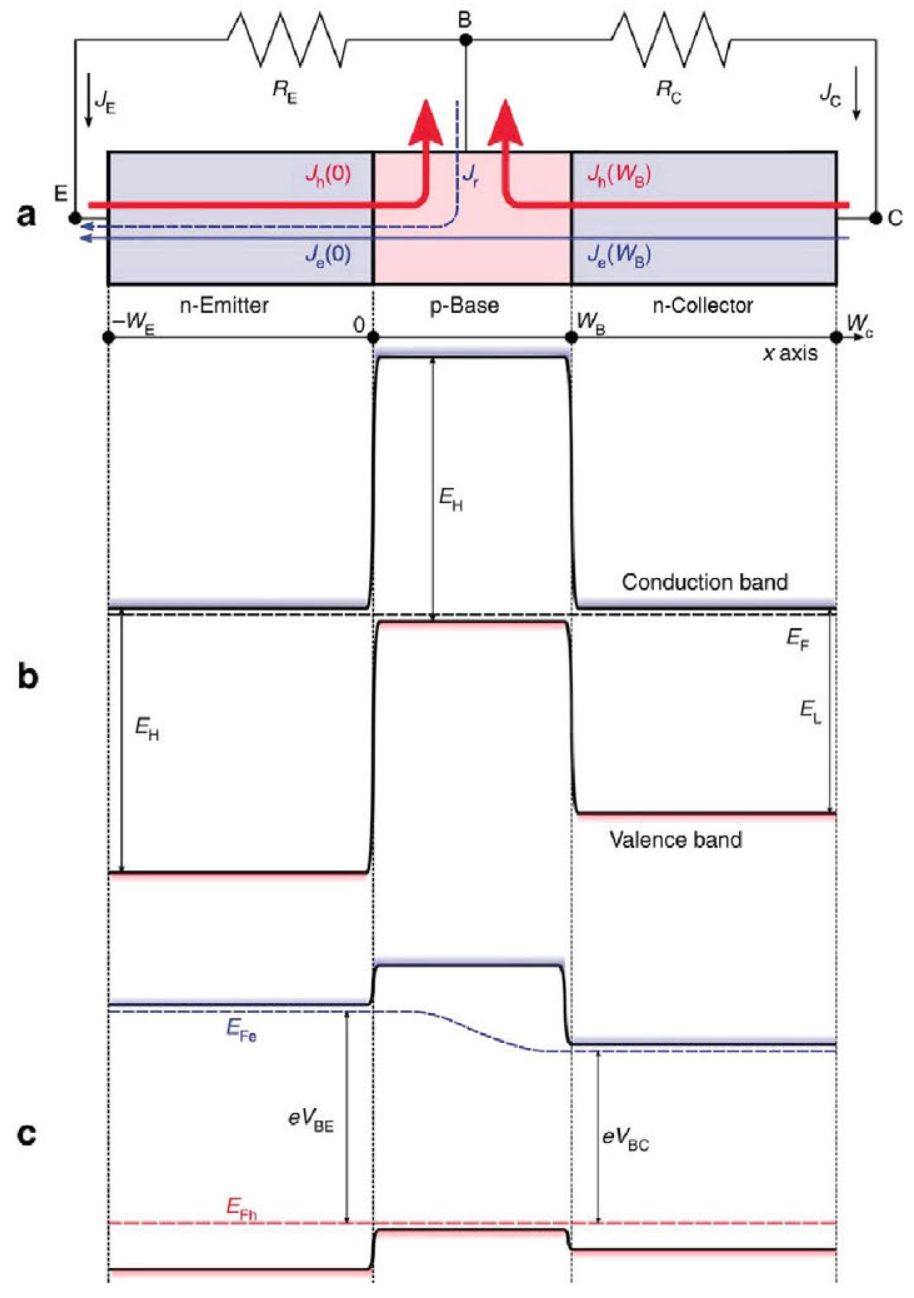

Fig. 1. Simplified layer structure of the HBTSC with electron and hole current densities $\left(J_{e}\right.$ and $\left.J_{h}\right)$. b) Simplified band diagram in equilibrium. c) Band diagram of the three-terminal HBTSC under working conditions. $E_{F e}$ and $E_{F h}$ are the quasi fermi levels for electrons and holes, respectively. $V_{B E}$ and $V_{B C}$ are the voltages of the top and bottom junction and $e$ is the electron charge. Adapted from: A. Martí and A. Luque, "Three-terminal heterojunction bipolar transistor solar cell for high-efficiency photovoltaic conversion" Nature Communications, vol. 6, p. 6902, 2015. http://www.nature.com/doifinder/10.1038/ncomms7902 (CC BY 4.0) 


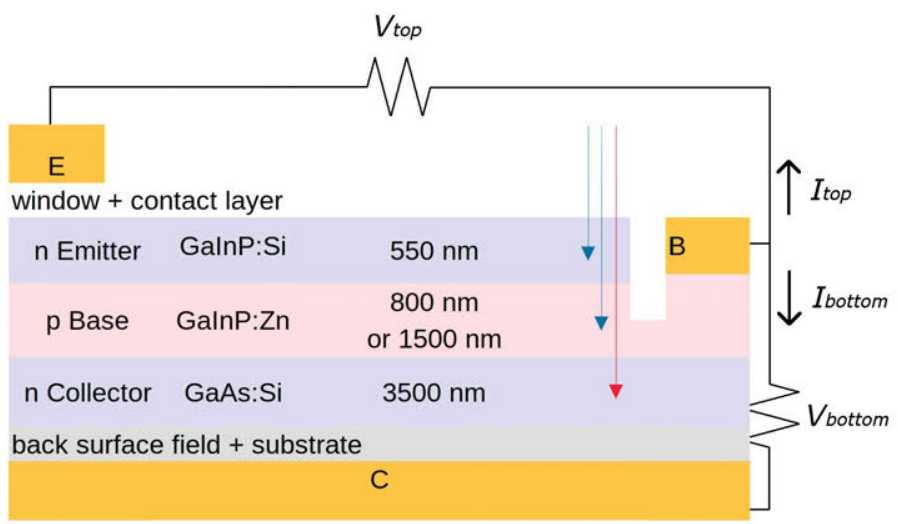

Fig. 2. Cell structure with metal contacts, labeled (E for emitter, B for base and $\mathrm{C}$ for collector). The circuit scheme for power extraction is included.

$p-n-p$ doped semiconductors. In this way, a double-junction solar cell is achieved with a very simple semiconductor layer structure, avoiding tunnel junctions. Fig. 1 shows the device structure and connection scheme (a) along with a simplified band structure of the cell in equilibrium (b) and under working conditions (c). The three-terminal nature of the cell allows for independent current extraction in both junctions, while an appropriate design makes it possible that the voltage across the top junction is higher than across the bottom junction.

Fig. 2 shows the structure and the three-terminal circuit of the HBTSC prototypes presented in this work. The main layers are called emitter, base and collector, like in a bipolar transistor. Emitter ( $n$-type) and base ( $p$-type) form the top junction, and the bottom junction is located between base and collector ( $n$-type). This results in a three-layer $n-p-n$ structure, comprising two functional $p-n$ junctions for photovoltaic conversion. The emitter has a front contact grid, while in the base an etching is required for contacting. The collector is contacted at the rear of the $n$-doped GaAs substrate, as in a conventional cell.

The most critical layer in the HBTSC design is the base, which must be capable of: (i) eliminating the transistor effect, that is, avoiding a voltage limitation in the top junction caused by the lower bandgap of the bottom junction [3], and (ii) to extract the current laterally without introducing too much series resistance $\left(R_{s}\right)$ (Fig. 2). For both purposes, studying the thickness of the base plays a fundamental role. We present two different cell designs, comparing a sample with a short base (SB), with the base thickness $\left(W_{B}\right)=800 \mathrm{~nm}$ and a sample with a long base (LB), with $W_{B}=1500 \mathrm{~nm}$.

The semiconductor structures were grown by metalorganic vapour-phase epitaxy at NREL and the cells processed at IESUPM (for details see section V). The prototypes provide a proof-of-concept for the GaInP/GaAs HBTSC with efficiencies $19.4 \%$ and $19.0 \%$ under AM1.5G spectrum without antireflection coating. The analysis of their performance provides a basis for future optimization of the HBTSC design.

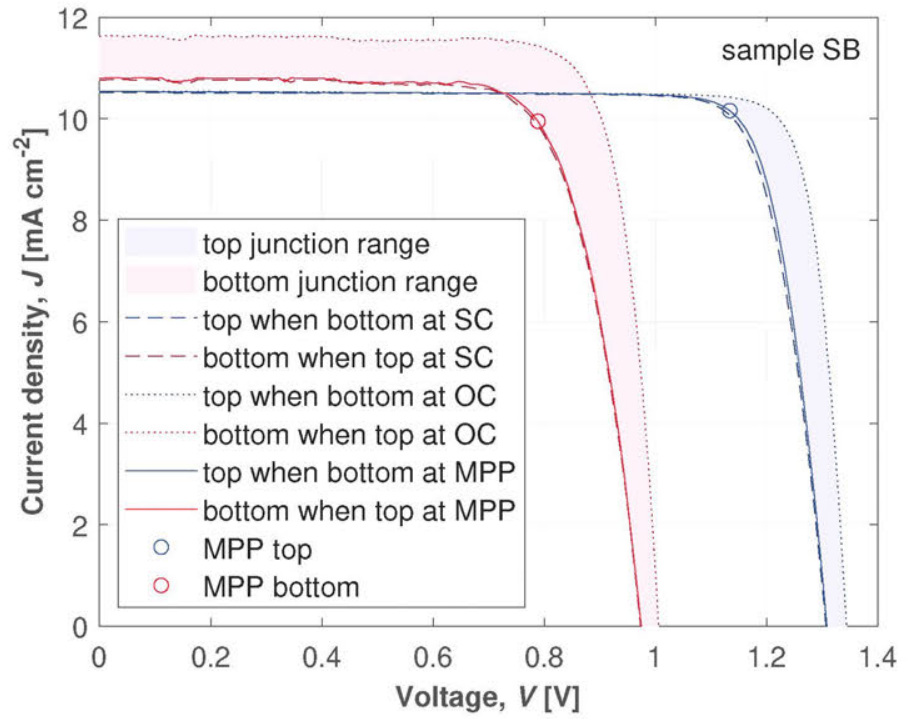

Fig. 3. Illuminated AM1.5G $J-V$ curves of sample SB. The curves of a junction depend on the working point of the other junction.

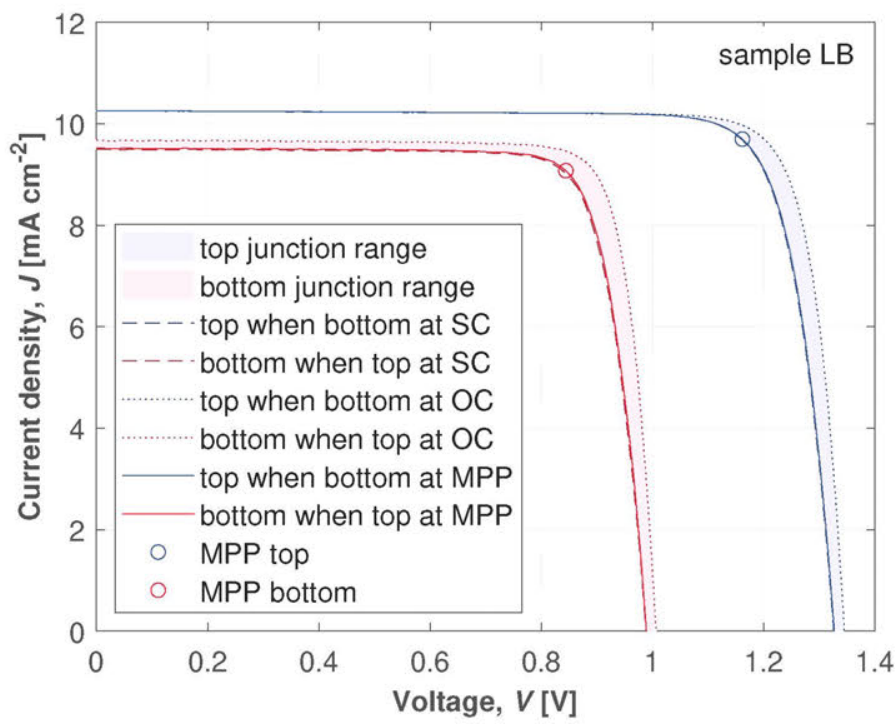

Fig. 4. Illuminated AM1.5G $J-V$ curves of sample LB. The curves of a junction depend on the working point of the other junction.

\section{EXPERIMENTAL RESULTS}

Figs. 3 and 4 show the illuminated current density $(J)$ voltage $(V)$ curves of both junctions in the two samples, depending on the working point of the other junction. They are similar to the $J-V$ curves of a conventional double-junction solar cell, in spite of the very simple transistor structure.

Because this is a three-terminal device and the sub-cells may interact, the definition of maximum power point (MPP) is not straightforward. The MPP of each sub-cell cannot be derived from its own $J$ - $V$ curve. If cross-talk between sub-cells is significant, it is necessary to add up the power produced by both junctions for a range of $\left(V_{b o t t o m}, V_{t o p}\right)$ values and find the maximum of that function. Therefore, 


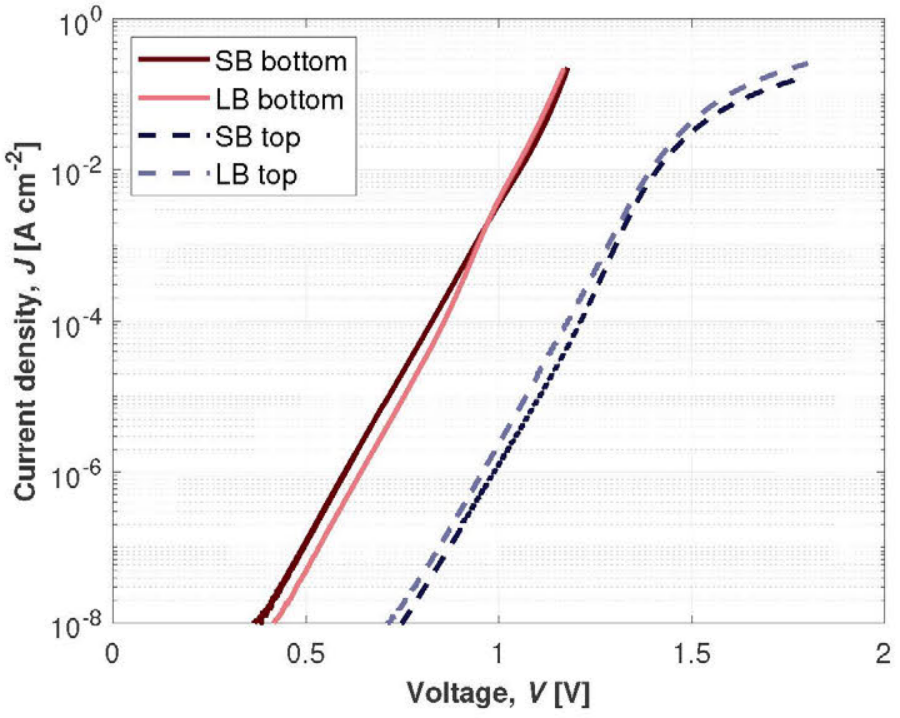

Fig. 5. Dark $J-V$ curves of each junction of the two cells. The curves are independent of the voltage bias if the junction that is not measured (for any voltage between zero and its one-sun $V_{O C}$ ).

throughout this paper, MPP refers to the working point that maximizes the total power produced by the device, which may not coincide with the $V$ value that maximizes the $J \cdot V$ product of a single junction as it would be extracted from any of the curves plotted in Figs. 3 and 4.

All junctions reach their maximum performance when the junction which is not being measured is at open circuit (OC), whereas biasing the other junction at its MPP or at short circuit (SC) decreases the performance. The top junctions are affected by a voltage drop: the $V_{O C}$ is higher when the bottom junctions are at $\mathrm{OC}$ and lower when the bottom junctions are at SC. The bottom junctions are affected by a similar voltage drop. Furthermore, the current of the bottom junctions is higher when the top junctions are biased at OC. In all junctions of both samples, the $J-V$ curves taken when the other junction is at its MPP is very similar to the ones taken when the other junction is at SC. The effects of the working point of the other junctions on the $J-V$ curves are smaller in sample LB than in sample SB.

Fig. 5 shows the dark $J-V$ curves of both cells. When measuring the dark $J-V$ curve of one junction, biasing the other junction in the range between $0 \mathrm{~V}$ and its one-sun $V_{O C}$ does not have a visible effect.

The external quantum efficiency (EQE) of both prototypes is plotted in Fig. 6. They are identical when measured with the other junction in $\mathrm{SC}$ or OC. The EQEs of both samples are similar in shape, although sample SB exhibits a lower $\mathrm{EQE}$, especially in the bottom junction.

Finally, Fig. 7 shows the $J-V$ curves under illumination. While measuring the $J-V$ curve of one junction, the other junction was biased at its MPP. The top junction of sample $S B$ reaches $1.31 \mathrm{~V}$ and the bottom junction $0.98 \mathrm{~V} V_{O C}$ under simulated one-sun illumination. Sample LB reaches $1.33 \mathrm{~V}$ and $0.99 \mathrm{~V}$, respectively. While the $V_{O C} S$ are slightly higher

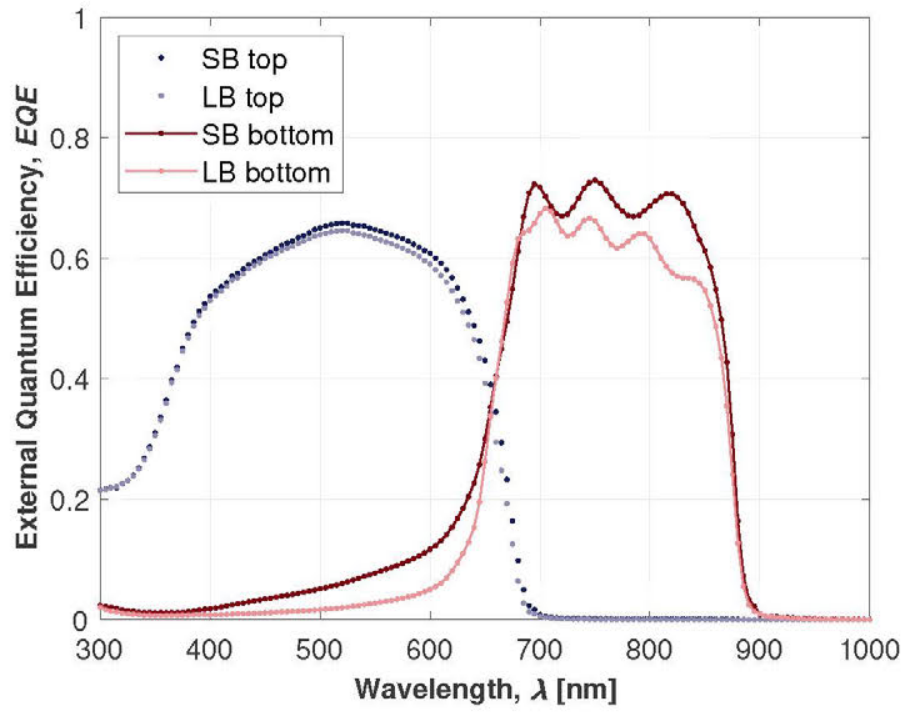

Fig. 6. External quantum efficiencies of samples SB and LB. The curves are independent of the voltage bias if the junction that is not measured (for any voltage between zero and its one-sun $V_{O C}$ ).

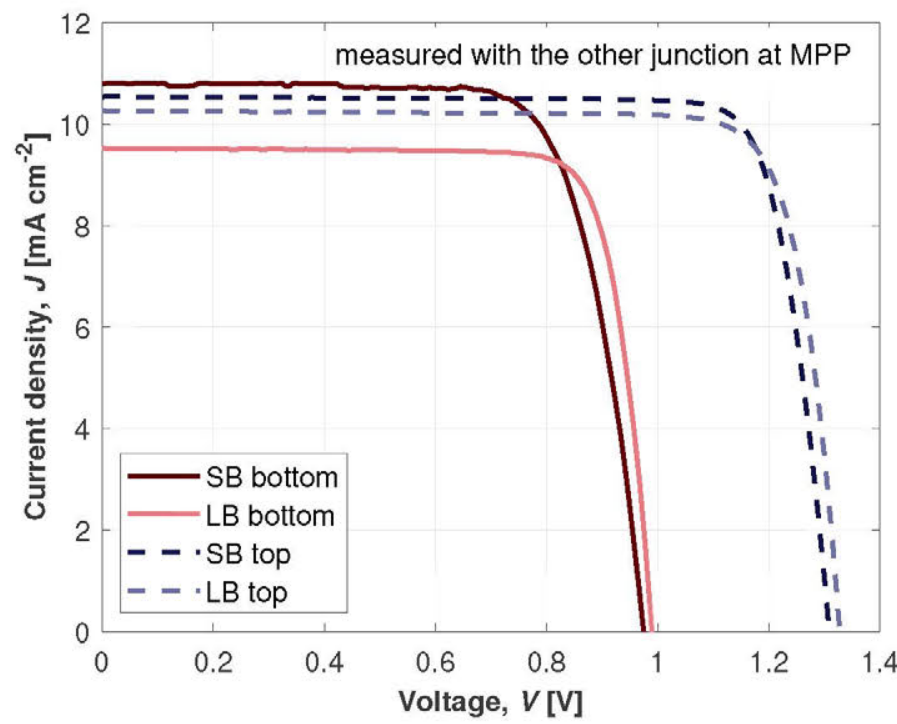

Fig. 7. Illuminated AM1.5G $J-V$ curves of samples SB and LB, measured with the other junction biased at MPP.

in sample $\mathrm{LB}$, the short-circuit currents $\left(J_{S C}\right)$ are lower. The parameters extracted from the curves presented in Figs. 5 and 7 are summarized in Table 1.

\section{DISCUSSION}

One of the critical aspects of the HBTSC design is the risk of a transistor effect between the junctions, which would make $V_{O C}$ of the top junction decrease and approach $V_{O C}$ of the bottom junction [3], [8]. The EQEs and $J-V$ curves in our case show that the prototypes do behave as a real doublejunction solar cell. However, we still see that lowering the bias of the bottom junction degrades the performance of the top junction. Let us examine this cross-talk between sub cells in more detail. 
TABLE 1

PARAMETERS OF THE AM1.5G AND DARK $J$ - $V$ CURVES.

THE ILLUMINATED CURVES WERE TAKEN WITH THE OTHER JUNCTION BIASED AT ITS MPP.

\begin{tabular}{|l|c|c|c|c|}
\hline Parameter & \multicolumn{2}{|c|}{ Bottom Junction } & \multicolumn{2}{|c|}{ Top junction } \\
Sample & SB & LB & SB & LB \\
\hline Illuminated area $\left[\mathrm{mm}^{2}\right]$ & 3.37 & 3.37 & 2.91 & 2.91 \\
$V_{O C}[\mathrm{~V}]$ & 0.98 & 0.99 & 1.31 & 1.33 \\
$J_{S C}\left[\mathrm{~mA} \mathrm{~cm}^{-2}\right]$ & 10.8 & 9.52 & 10.5 & 10.3 \\
$P_{M P P}\left[\mathrm{~mW} \mathrm{~cm}^{-2}\right]$ & 7.9 & 7.7 & 11.5 & 11.3 \\
$F F$ & 75 & 81 & 84 & 83 \\
$\eta[\%]$ & 7.9 & 7.7 & 11.5 & 11.3 \\
\hline Dark area $\left[\mathrm{mm}^{2}\right]$ & 4.0 & 4.0 & 3.3 & 3.3 \\
$J_{01}[\mathrm{Acm}-2]$ & $1 \cdot 10^{-20}$ & $7 \cdot 10^{-20}$ & $8 \cdot 10^{-21}$ & $8 \cdot 10^{-21}$ \\
$m_{1}$ & 1.0 & 1.0 & 1.3 & 1.3 \\
$J_{02}\left[\mathrm{Acm} \mathrm{cm}^{-2}\right]$ & $2.3 \cdot 10^{-12}$ & $7.0 \cdot 10^{-13}$ & $5.0 \cdot 10^{-15}$ & $1.0 \cdot 10^{-14}$ \\
$m_{2}$ & 1.8 & 1.8 & 2.0 & 2.0 \\
$R_{s}(\mathrm{dark})\left[\Omega \mathrm{cm}^{2}\right]$ & - & - & 1.8 & 1.1 \\
$R_{p}\left[\Omega \mathrm{cm}^{2}\right]$ & $>1 \cdot 10^{9}$ & $>9 \cdot 10^{8}$ & $>1 \cdot 10^{9}$ & $>1 \cdot 10^{9}$ \\
\hline
\end{tabular}

We begin with the effect on the current of the junctions: when the top junctions are biased at $V_{O C}$, the $J_{S C}$ s of the bottom junctions are higher. However, this does not occur in the opposite direction. Thus, we identify luminescent coupling [9] as the cause.

Now we focus on the voltage drop produced in the illuminated $J-V$ curves when the junction which is not being measured is in SC. This could be an indication of a transistor effect, but it could also be caused by resistive losses in the base layer. In a conventional solar cell, series resistance $\left(R_{s}\right)$ has no effect in open-circuit because there is no current flow. However, it can have an effect in a three-terminal device: in our case, the photocurrent generated by a junction flows through the base and produces a voltage drop that will affect the $J-V$ of the other junction at any working point, including its $V_{O C}$

Note that in our design the $R_{s}$ loss produced in the base can be large because current flows laterally under illumination (Fig. 2). It is expected to be larger in sample SB than in sample LB due to the difference in $W_{B}$. From the fitting of the dark $J-V$ curves we estimate that $R_{s}$ is $1.8 \Omega \mathrm{cm}^{2}$ and $1.1 \Omega \mathrm{cm}^{2}$ for samples SB and LB respectively (Table 1 ), and our calculations indicate that those $R_{s}$ values can be entirely attributed to the lateral transport in the base. Therefore, the voltage drop caused by $R_{s}$ in either junction of sample SB when the other junction goes from $\mathrm{OC}$ to $\mathrm{SC}$ is expected to be $\sim 20 \mathrm{mV}$ for $\mathrm{SB}$ and $\sim 10 \mathrm{mV}$ in the case of $\mathrm{LB}$. These values are comparable, within the voltage and temperature precision of illuminated $J-V$ curves, to the voltage drops observed in Figs. 3 and 4.

This indicates that the voltage drop is related to resistive losses and there is virtually no cross-talk between sub-cells due to the transistor effect (under this illumination level). The fact that we observe no cross-talk between sub-cells when they are in the dark (Fig. 5) corroborates our interpretation, since there is no current flow across the short-circuited junction in that case. From this analysis it is concluded that the strategy of increasing $W_{B}$ is advantageous for our prototypes because it limits the $V_{O C}$ and $F F$ degradation of resistive nature.

On the other hand, we see that $J_{S C}$ S are lower for LB than for SB, especially those of the bottom junction (Figs. 6 and 7; Table 1). Although it could be anticipated that this is caused by the lack of transparency of the base in LB, the EQEs (Fig. 6) reveal that the photogeneration at long wavelengths has decreased too. Also, sample LB shows a lower contribution of luminescent coupling to the photocurrent of the bottom sub-cell (Figs. 3 and 4). These observations point to a lower material quality in sample LB. A plausible explanation is the diffusion of $\mathrm{Zn}$ from the base to the collector during growth which is to be expected due to the high doping concentration required in the base.

Fig. 8 shows a secondary ion mass spectroscopy of the dopant atoms of a sample grown with the same parameters as our samples, and slightly different layer thicknesses. The zinc concentration has a high peak at the base-collector interface, which is indicative of $\mathrm{Zn}$ diffusion. A longer base requires a longer growth and therefore in sample LB Zn diffusion will affect the material quality more than in sample SB.

This effect on the photocurrent counteracts the improvement produced by the reduction of series resistance losses, resulting in a marginally lower efficiency for the longbase sample compared to the short-base sample. In future GaInP/GaAs HBTSC prototypes the structure will have to be optimized to solve this trade-off. Also, applying interdigitated metal masks for the emitter and base contact would help reduce the $R_{s}$ of the base without increasing its thickness.

\section{CONCLUSIONS}

We show the first HBTSC made of a heterojunction of GaInP and GaAs. The voltage difference between the two junctions is remarkable taking into account that this is a thin film device and both junctions share a $0.8 \mu \mathrm{m}$ layer in sample SB. The fill factors are above $80 \%$ for both junctions 


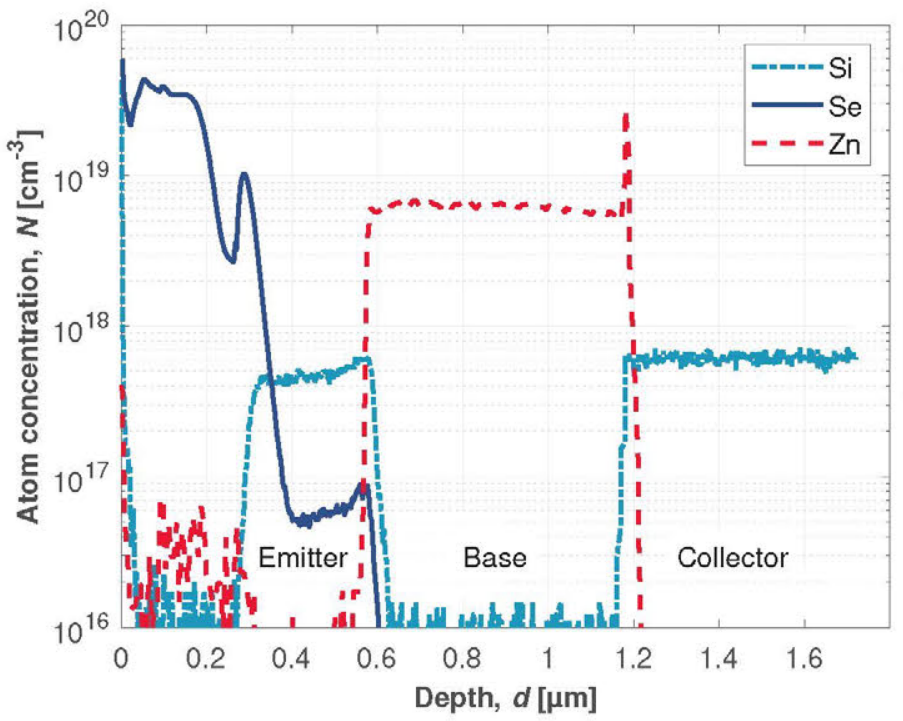

Fig. 8. Dopant atom concentration in the semiconductor material as a function of depth from the surface as extracted from SIMS measurements. This measurement was taken of another sample, with layer thicknesses differing from the ones in Table (1), but grown with the same reactor and parameters.

in sample LB and the $V_{O C} \mathrm{~S}$ are comparable to monolithic series-connected double-junction record cells. It has to be noted that this device has a total of six layers (with no tunnel junction) and state-of-the-art series-connected doublejunction cells have at least 13 [4].

Our results indicate that this HBTSC structure is a basis to create a highly efficient double-junction solar cell which combines a very simple and compact structure with the potential of achieving high annual energy yield thanks to the three-terminal configuration.

At one-sun illumination, reducing one junction's bias from OC to MPP degrades the performance of the other junction only slightly $(<0.5 \%$ efficiency loss). Our analysis shows that this loss is related to $R_{s}$ and luminescent coupling effects rather than to an intrinsic limitation of the transistor structure.

The fact that the contribution of the base to $R_{s}$ is affecting both junctions at the same time makes the $J-V$ curves of a junction drop to lower voltages when the other junction has a higher current. It is crucial to keep $R_{s}$ low, particularly in the base layer, for high performance HBTSCs. An interdigitated cell design might help in overcoming this problem.

\section{METHODS}

We presented the results of an HBTSC made of gallium indium phosphide (GaInP) and gallium arsenide (GaAs). Two samples, which differ in the thickness of one layer, were compared. The semiconductor structures have been grown by metal-organic vapor-phase epitaxy (MOVPE) at NREL and devices have been processed using standard photolithography, wet etching and metal evaporation techniques at IES-UPM.

Table (2) shows the detailed semiconductor structures, including contact, window and BSF layers. The samples are
TABLE 2

SOLAR CELL STRUCTURES

\begin{tabular}{|l|c|c|l|c|}
\hline $\begin{array}{l}\text { Sample } \\
\text { Layer }\end{array}$ & $\begin{array}{c}\text { Short Base } \\
\text { Thickness }[\mathbf{n m}]\end{array}$ & $\begin{array}{c}\text { Long Base } \\
\text { Material }\end{array}$ & $\begin{array}{c}\text { Doping } \\
{\left[\mathrm{cm}^{-2}\right]}\end{array}$ \\
\hline Contact & 280 & 250 & $n$-GaAs & $\mathrm{Se}$ \\
Window & 25 & 25 & $n$-AlInP & $\mathrm{Se}$ \\
Emitter & 550 & 550 & $n$-GaInP & $\mathrm{Si} 6 \cdot 10^{17}$ \\
Base & 800 & 1500 & $p$-GaInP & $\mathrm{Zn} 7 \cdot 10^{18}$ \\
Collector & 3500 & 3500 & $n$-GaAs & $\mathrm{Si} 6 \cdot 10^{17}$ \\
BSF & 300 & 300 & $n$-GaInP & $\mathrm{Si}$ \\
Substrate & & & $n$-GaAs & $\mathrm{Si}$ \\
\hline
\end{tabular}

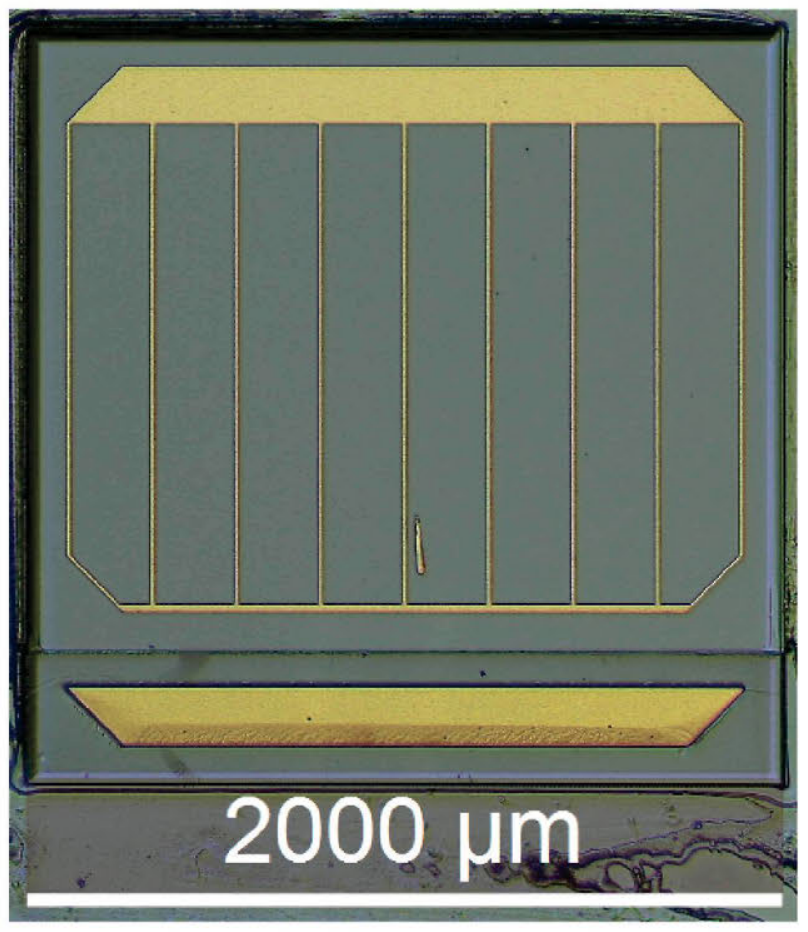

Fig. 9. Micrograph of processed cell.

nominally identical, except for $W_{B}$. The dopant concentrations of the different layers, taken from a similar sample, are given in the secondary ion mass spectroscopy (SIMS) measurements shown in Fig. 8.

The processing can be summarized in the following steps: For the base contact, $15 \mathrm{~nm} \mathrm{Au} / 40 \mathrm{~nm} \mathrm{Zn} \mathrm{/} 500 \mathrm{~nm} \mathrm{Au}$ were thermally evaporated [10]. The emitter contact was made of $85 \mathrm{~nm} \mathrm{AuGe} / 25 \mathrm{~nm}$ Ni / $500 \mathrm{~nm}$ Au [11]. Finally, the collector contact was made of $85 \mathrm{~nm}$ AuGe / $25 \mathrm{~nm} \mathrm{Ni} /$ $300 \mathrm{~nm}$ Au. All contacts were annealed simultaneously at $420^{\circ} \mathrm{C}$ for $180 \mathrm{~s}$ in forming gas $\left(5 \% \mathrm{H}_{2}\right.$ in $\left.\mathrm{N}_{2}\right)$. Nonselective controlled wet etching of GaInP (emitter and base) was done by $\mathrm{HCl} 12 \mathrm{M}$ in $\mathrm{H}_{2} \mathrm{O}$ at $3^{\circ} \mathrm{C}$ with a rate of up to $12 \mathrm{nms}^{-1}$. For mesa isolation, $\mathrm{HCl}: \mathrm{CH}_{3} \mathrm{COOH}: \mathrm{H}_{2} \mathrm{O}_{2}$ 2:4:3 as nonselective etch for GaInP and GaAs was used [12]. Fig. 9 shows a micrograph of a processed sample LB. Etching of $\mathrm{GaInP}$ with $\mathrm{HCl}$ is problematic because of its anisotropy [13] We incorporated tolerances of $100 \mu \mathrm{m}$ between etched areas and metalization to avoid device failure. After processing, the cells were mounted on a copper disk and contacted by wire 
bonding for measurements.

The dark $J-V$ curves were fitted to a two diode model, permitting $m_{1} \neq 1$ for the top junctions. In these curves, the dark junction areas were considered.

For the illuminated measurements, the solar simulator was spectrally matched to reach the light current calculated from the external quantum efficiency (Fig. 6) at an incident spectrum of AM1.5G $\left(100 \mathrm{~mW} \mathrm{~cm}^{-2}\right)$ for both junctions simultaneously. The illuminated area of each junction was considered for EQEs, illuminated current densities and efficiencies.

\section{ACKNOWLEDGMENT}

We are grateful to MADRID-PV2-CM financed by the Madrid Regional Government (Comunidad de Madrid) Grant S2018/EMT-4308 supported by FEDER funds. I.G., E.A. and P.G-L acknowledge the funding from Universidad Politécnica de Madrid through "Programa Propio para Ayudas para Fortalecer Planes de Investigacion" 2018-2019. M.H.Z. is grateful to the Universidad Politécnica de Madrid for the funding from the 'Programa Propio para Ayudas Predoctorales de 1a UPM'. I. G. and E. A. are funded by Ramón y Cajal Fellowships by the Spanish Ministry of Science (RYC-201415621 and RYC-2015-18539, respectively). This work was supported by the National Renewable Energy Laboratory for the U.S. Department of Energy (DOE) under Contract No. DE-AC36-08GO28308. Funding provided by the U.S. Department of Energy, Office of Energy Efficiency and Renewable Energy, Solar Energy Technologies Office (SETO). The views expressed in the article do not necessarily represent the views of the DOE or the U.S. Government.

\section{REFERENCES}

[1] A. S. Brown and M. A. Green, "Limiting efficiency for currentconstrained two-terminal tandem cell stacks," Prog. Photovoltaics, vol. 10, no. 5, pp. 299-307, Aug. 2002.

[2] J. Villa and A. Martí, "Impact of the Spectrum in the Annual Energy Production of Multijunction Solar Cells," IEEE Journal of Photovoltaics, vol. 7, no. 5, pp. 1479-1484, Sep. 2017. [Online]. Available: http://ieeexplore.ieee.org/document/7962291/
[3] A. Martí and A. Luque, "Three-terminal heterojunction bipolar transistor solar cell for high-efficiency photovoltaic conversion," Nature Communications, vol. 6, p. 6902, Apr. 2015. [Online]. Available: http:/Www,nature,com/doifinder/10.1038/ncomms 7902

[4] I. García, I. Rey-Stolle, B. Galiana, and C. Algora, "A 32.6\% efficient lattice-matched dual-junction solar cell working at 1000 suns," Applied Physics Letters, vol. 94, no. 5, p. 053509, Feb. 2009. [Online]. Available: http://aip, scitation,org/doi/10.1063/1.3078817

[5] S. Essig, C. Allebé, T. Remo, J. F. Geisz, M. A. Steiner, K. Horowitz, L. Barraud, J. S. Ward, M. Schnabel, A. Descoeudres, D. Young, M. Woodhouse, M. Despeisse, C. Ballif, and A. Tamboli, "Raising the one-sun conversion efficiency of III-V/Si solar cells to $32.8 \%$ for two junctions and $35.9 \%$ for three junctions," Nature Energy, vol, 2, no. 9, p. 17144, Aug. 2017. [Online]. Available: http://www.nature.com/articles/nenergy2017144

[6] M. Schnabel, M. Rienacker, E. L. Warren, J. F. Geisz, R. Peibst, P. Stradins, and A. C. Tamboli, "Equivalent Performance in ThreeTerminal and Four-Terminal Tandem Solar Cells," IEEE Journal of Photovoltaics, vol. 8, no. 6, pp. 1584-1589, Nov, 2018. [Online]. Available: https://ieexplore.ieee.org/document/8454791/

[7] H. Schulte-Huxel, T. J. Silverman, M. G. Deceglie, D. J. Friedman, and A. C. Tamboli, "Energy Yield Analysis of Multiterminal Si-Based Tandem Solar Cells," IEEE Journal of Photovoltaics, vol. 8, no. 5, pp. 1376-1383, Sep. 2018. [Online]. Available: https://ieeexplore.ieee.org/document/8405753/

[8] A. Martí, E. Antolín, P. García-Linares, E. López, J. Villa, and I. Ramiro, "Operation of the Three Terminal Heterojunction Bipolar Transistor Solar Cell," physica status solidi c, vol. 14, no. 10, p. 1700191, 2017. [Online]. Available: https://onlinelibrary.wiley.com/ doi/abs/10.1002/pssc.201700191

[9] M. A. Steiner and J. F. Geisz, "Non-linear luminescent coupling in series-connected multijunction solar cells," Appl. Phys. Lett., vol. 100, no. 25, p. 251106, Jun. 2012. [Online]. Available: https://aip.scitation.org/doi/abs/10.1063/1.4729827

[10] T. Sanada and O. Wada, "Ohmic Contacts to p-GaAs with $\mathrm{Au} / \mathrm{Zn} / \mathrm{Au}$ Structure," Japanese Journal of Applied Physics, vol. 19, no. 8, pp. L491-L494, Aug. 1980. [Online]. Available: http: //stacks.iop.org/1347-4065/19/L491

[11] R. Bruce and G. Piercy, "An improved Au-Ge-Ni ohmic contact to n-type GaAs," Solid-State Electronics, vol. 30, no. 7, pp. 729-737, Jul. 1987. [Online], Available: http://linkinghub.elsevier.com/retrieve/ pii/0038110187901122

[12] J. R. Flemish, "Selective Wet Etching of GaInP, GaAs, and InP in Solutions of $\mathrm{HCl}, \mathrm{CH}_{3} \mathrm{COOH}$, and $\mathrm{H}_{2} \mathrm{O}_{2}$, " Journal of The Electrochemical Society, vol. 140, no. 3, p. 844, 1993. [Online]. Available: http://jes.ecsdl.org/cgi/doi/10.1149/1.2056170

[13] M. J. Cich, J. A. Johnson, G. M. Peake, and O. B. Spahn, "Crystallographic dependence of the lateral undercut wet etching rate of InGaP in $\mathrm{HCl}$," Applied Physics Letters, vol. 82, no. 4, pp. 651-653, Jan. 2003. [Online]. Available: http://aip.scitation.org/doi/10. $1063 / 1,1540236$ 